\title{
EVALUATION OF AMBULATORY ARTERIAL STIFFNESS INDEX IN HYPERTHYROIDISM
}

Ozlem Turhan Iyidir ${ }^{1}$, Muhittin Yalcin, ${ }^{1}$ Alev Eroglu Altinova ${ }^{1}$, Emre Arslan ${ }^{1}$ Bahar Uncu ${ }^{2}$, Ceyla Konca Degertekin ${ }^{1}$, Fusun Balos Toruner ${ }^{1}$

1 : Gazi University, Department of Endocrinology and Metabolism

2 : Gazi University, Department of Internal Medicine

\section{BACKGROUND}

Hyperthyroidism, whether endogenous or exogenous in origin, causes hemodynamic changes that are associated with adverse cardiovascular outcomesAmbulatory arterial stiffness index (AASI) is a non-invasive way of measuring arterial stiffness and defined as 1 minus the regression slope of diastolic on systolic BP values derived from a 24-hour ambulatory blood pressure monitoring (ABPM) recordings. Several studies showed an association betweeen overt and subclinical hyperthyroidism and increased arterial stiffness, as well as impaired vascular elasticity. However, there is no data about AASI and its relationship with thyroid hormones. In this study, we aimed to investigate AASI and the BP variability as the indicators of cardiovascular risk in overt and subclinical hyperthyroidism as well as healthy control subjects.

\section{METHODS}

We enrolled 59 patients with hyperthyroidism and 25 healthy euthyroid controls in the study. The hyperthyroid group included 36 patients with subclinical hyperthyroidism and 23 patients with overt hyperthyroidism. Patients with both endogenous and exogenous subclinical hyperthyroidism were included in the study. ABPM recording was performed for 24-hours using Spacelabs model 90207 monitors (Issaquah, WA, USA), on a day of standard activity, with an adequate cuff size of the patient's arm. The records of readings considered to be valid were $\geq 80 \%$ of the total. Systolic and diastolic BP variability were expressed by the coefficient of variation (CV) of systolic or diastolic measurements, defined by using relative changes $(\mathrm{CV}=100 \mathrm{x}$ s.d./mean). AASI, is defined as 1-(diastolic-on-systolic slope), wherein the slope was determined from a DBP vs. SBP plot by a standard regression procedure.

\section{RESULTS}

The hyperthyroid group included 36 patients with subclinical hyperthyroidism and 23 patients with overt hyperthyroidism. There were no statistically significant differences among overt hyperthyroidism, subclinical hyperthyroidism and control groups in terms of AASI ( $0.43 \pm 0.15$, $0.38 \pm 0.12,0.42 \pm 0.13$, respectively; $\mathrm{p}=0.315)$. Variability of diastolic $\mathrm{BP}$, as expressed by 24-hour diastolic coefficient variation (CV), was significantly higher in patients with overt hyperthyroidism than patients with subclinical hyperthyroidism $(14.8 \pm 2.6 \mathrm{vs} 12.8 \pm 2.5 \%$, $\mathrm{p}=0.023)$. There were significant positive correlations between AASI and fT3 $(r=0.246, p=0.02)$ and fT4 $(r=0.219, p=0.04)$ while TSH was not correlated with AASI $(\mathrm{r}=0.023, \mathrm{p}=0.838)$. After adjusting for confounders, age, 24-hour systolic and diastolic BP, variability of systolic and diastolic BP (24-hour systolic and diastolic CV) and fT4 were independent predictors of AASI $(r 2=0.460, \mathrm{p}<0.001)$

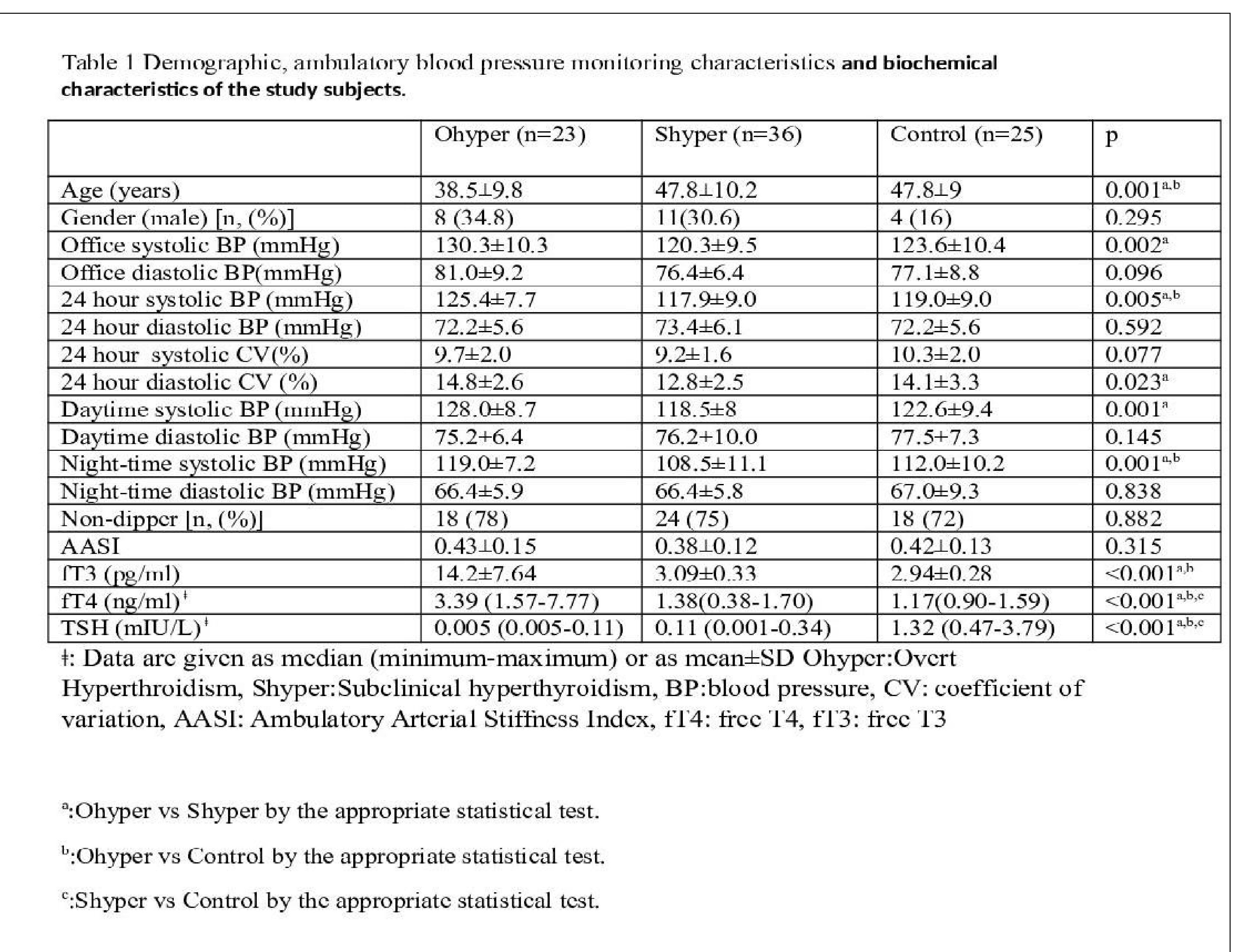

Table 2 Independent Predictors of AASI in a Multiple Regression Analysis best fitting model
$\left(\mathrm{R}^{2}=0.460\right)$
\begin{tabular}{|l|l|l|}
\hline & $\beta$ & $\mathrm{p}$ \\
\hline Age (years) & 0.003 & 0.044 \\
\hline Gender & 0.039 & 0.182 \\
\hline 24 hour Systolic BP (mmHg) & 0.008 & $<0.001$ \\
\hline 24 hour diastolic BP (mmHg) & -0.012 & $<0.001$ \\
\hline 24 hour systolic CV(\%) & 0.040 & 0.001 \\
\hline 24 hour diastolic CV $(\%)$ & -0.044 & $<0.001$ \\
\hline Non-dipper & -0.009 & 0.758 \\
\hline fT4 (ng/ml) & 0.020 & 0.045 \\
\hline
\end{tabular} BP:blood pressure CV: coefficient of variation, AASI: Ambulatory Arterial Stiffness Index
fT4: free T4

\section{SUMMARY OF CONCLUSIONS}

In conclusion, our study showed that AASI did not differ between overt and subclinical hyperthyroidism whereas short term BP variability was higher in overt hyperthyroidism than subclinical hyperthyroidism. Also, there was a positive relationship between AASI and free thyroid hormones. Further studies are needed to enlighten the possible relation between arterial stiffness and excess thyroid hormones. Larger prospective studies investigating the alteration in BP variability and AASI after maintaining euthyroidism may potentially broaden our understandings about vascular effects of thyroid hormones

\section{REFERENCES}

1. S. Danzi, I. Klein. Thyroid disease and the cardiovascular system. Endocrinol Metab Clin North Am, 2014. 43(2): p. 517-28

2. G. Schillaci, G. Parati. Ambulatory arterial stiffness index: merits and limitations of a simple surrogate measure of arterial compliance. J Hypertens, 2008. 26(2): p. 182-5.

3. J. Bahrainwala, A. Patel, K.M. Diaz, et al. Ambulatory Arterial Stiffness Index and circadian blood pressure variability. J Am Soc Hypertens, 2015. 9(9): p. 705-10.

4. P. Iglesias, M. Acosta, R. Sanchez, et al. Ambulatory blood pressure monitoring in patients with hyperthyroidism before and after control of thyroid function. Clin Endocrinol (Oxf), 2005. 63(1): p. 66-72. 